\title{
Strain-driven diffusion process during silicon oxidation investigated by coupling Density Functional Theory and Activation Relaxation Technique
}

\author{
N. Salles ${ }^{1}$, N. Richard ${ }^{2}$, N. Mousseau ${ }^{3}$ and A. Hemeryck ${ }^{1 *}$ \\ ${ }^{1}$ LAAS CNRS, Université de Toulouse, CNRS, Toulouse, France \\ ${ }^{2}$ CEA, DAM, DIF, Bruyères-le-Châtel, F-91297 Arpajon, France \\ ${ }^{3}$ Université de Montréal, Département de Physique \\ C.P. 6128, succursale Centre-ville H3C 3J7 Montréal Canada
}

\begin{abstract}
The reaction of oxygen molecules on an oxidized silicon model-substrate is investigated using an efficient potential energy hypersurface exploration that provides a rich picture of the associated energy landscape, energy barriers and insertion mechanisms. Oxygen molecules are brought in, one by one, onto an oxidized silicon substrate and accurate pathways for sublayer oxidation are identified through the coupling of Density Functional Theory to the Activation Relaxation Technique nouveau, an open-ended unbiased reaction pathway searching method, allowing full exploration of potential energy surface.

We show that strain energy increases with $\mathrm{O}$ coverage, driving the kinetics of diffusion at the $\mathrm{Si} / \mathrm{SiO}_{2}$ interface in the interfacial layer and deeper into the bulk: at low coverage, interface reconstruction dominates while at high coverage oxygen diffusion at the interface or even deeper into the bottom layers is favored. A changing trend in energetics is observed that favors atomic diffusions to occur at high coverage while they appear to be unlikely at low coverage. Upon increasing coverage, strain is accumulated at the interface, allowing the oxygen atom to diffuse as the strain becomes large enough. The observed atomic diffusion at the interface releases the accumulated strain, which is consistent with a layer-by-layer oxidation growth.
\end{abstract}

\section{Corresponding author:}

*Anne HEMERYCK, LAAS-CNRS, 7 avenue du Colonel Roche, BP 54200, 31031 Toulouse Cédex 4, email: anne.hemeryck@laas.fr 


\section{INTRODUCTION}

For more than fifty years, silicon $(\mathrm{Si})$ and its oxide, silica $\left(\mathrm{SiO}_{2}\right)$, have been the materials of choice in the field of microelectronics notably because of the remarkable quality of its interface in which $\mathrm{SiO}_{2}$ serves as gate oxide on the silicon substrate into Metal Oxide Semiconductor Field-Effect Transistors (MOSFETs) [1]. More recently, this interface has also been used in Metal-Insulator-Semiconductor (MIS) nanojunctions [2] and in silicon spintronics [3] among other applications. Moreover, in many applicative systems, the growth of a different oxide layer on a silicon substrate first requires the preparation of a thin silica layer between the silicon and the oxide. A total control of the growth, the thickness and the quality of this layer is then mandatory in order to obtain the desired properties for the components as illustrated by the case of hafnia based MOSFETS [4]. From a more fundamental point of view, this interface is a model system for growing an oxide on a semiconductor, but also for the formation of an amorphous from a crystalline system. Yet, despite intensive experimental [5-12 and references therein] and theoretical studies [13-24], many fundamental questions about the basic mechanisms of the early formation stages of this crystalline/amorphous interface, including the role of strain-driven reactions, remain unresolved. Strain effect appears to be at the origin of the emission of interstitial silicon atom at the interface allowing the transition from a dense silicon substrate to an amorphous oxide $[8,9,25,26]$. A layer-by-layer growth mode as observed in silicon thermal oxidation has also been attributed to a strain-driven propagation of the oxidation front [25-28]. A relation between oxidation coverage, temperature dependency and interfacial strain has been established $[8,29,30]$. More recently, a retarded or self-limiting oxidation of silicon nanowires has been also attributed to mechanical effects [31-33]. A comprehensive atomic-scale view of silica growth is still lacking.

To advance on this issue, we follow an innovative approach: starting from previously calculated configurations for the first oxidized layer, we use the Activation Relaxation Technique (ART nouveau or ARTn) [34-36] coupled with first principles calculations using the VASP package [37,38]. The choice of Density Functional Theory (DFT) based calculations allows us to describe precisely all the atomic and electronic structure variations associated with oxidation such as spin transition and charge transfer.

For up to a monolayer of oxide coverage, it is still possible to explore the configurational space by hand. However, as oxygen molecules are further added, the number of possible configurations and diffusion paths in the subsurface and deeper into the bulk, crucial to the 
growth of the oxidized layer, rapidly increases, involving complex mechanisms associated with large rearrangements and the displacement of several atoms. In such cases, double-ended methods to determine minimum energy pathways, such as the Nudged Elastic Band method, are insufficient as they require a pre-knowledge or a guess of the final configuration. It is necessary to turn, rather, to open-ended search methods such as the Activation Relaxation Technique nouveau (ARTn) [34-36], Eigenvector Following (EF) [39] or the Dimer method [40], that allow the unbiased exploration of space configurations and diffusion mechanisms. For silicon oxidation, ARTn seems well fitted to the problems we address. As an open-ended saddle point search algorithm, this algorithm has already clearly demonstrated its efficiency to explore a set of configurations even in complex disordered systems and defect diffusion $[41,42]$. Furthermore, it has already been used for a systematic study on silicon oxidation using empirical potentials showing its applicability to this issue by performing the diffusion and insertion of the oxygen atoms into the silicon-silicon bonds and supplying the energy barriers between the various generated configurations [43].

At the initial stage, Si (100) oxidation grows layer-by-layer [5,6] and the oxidation rate change is strongly correlated with the amount of strained $\mathrm{Si}$ at the interface [8]. These experimental results suggest a $\mathrm{Si}$ oxidation model mediated by point defect generation (emitted $\mathrm{Si}$ atoms and their vacancies) due to the oxidation-induced strain [7-9]. The aim of this article is to develop a detailed atomic-scale understanding of the propagation of the oxidation rate starting from a simple oxidized model system, with a particular focus on the correlation between the strain at the interface and the oxidation propagation rate, an aspect that is very difficult to characterize experimentally. To do so, we start from configurations observed in previous DFT studies $[8,9,10,16,17,43,44]$ on the first monolayer oxidation. Oxygen coverage is then increased and oxygen adsorption and further diffusion at the $\mathrm{Si} / \mathrm{SiO}_{2}$ interface is studied using VASP-ARTn coupling. This approach allows us to establish a direct link between the subsurface diffusion mechanism in close relation with the strain energy and its effect on the energetics in terms of kinetics and energy gains.

\section{METHODOLOGY}

\subsection{ART nouveau}

The Activation Relaxation Technique nouveau (ARTn) method allows an efficient and unbiased search of possible migration paths for an atom in a given system. ARTn explores the potential energy surface (PES) and determines possible transition states, i.e. the different 
configurations and their corresponding activation barriers from a given starting configuration, without the need for prior knowledge of the final states.

ARTn proceeds in three steps: (1) From a local energy minimum, a random deformation is applied in order to leave the harmonic basin. As the system is slow deformed, the structure is partially relaxed in hyperplane orthogonal to this displacement and the lowest eigenvalue of the Hessian matrix is calculated with 10 to 15 force evaluations using Lanczos algorithm. (2) Once a negative eigenvalue is found, the configuration is pushed up along the corresponding eigenvector while forces are minimized in the perpendicular hyperplane. This procedure brings the structure to the associated first-order saddle point, converging with any desired precision. (3) When the saddle point is reached, a step of energy minimization is performed in order to bring the configuration into a new minimum.

\subsection{Coupling ART nouveau and DFT}

Until now, ARTn has been mostly employed with empirical potentials or local-basis ab initio codes such as SIESTA [39] and BigDFT [36]. For the present study, such level of description is insufficient since known empirical potentials cannot take into account the complex chemistry of oxidation reaction and associated rearrangement. We therefore select to use the plane-wave DFT VASP [37,38] code and interfaced this widely used package directly with the ARTn code.

ARTn code finds a saddle point around a local minimum by following the eigenvector associated at one negative eigenvalue of the hessian matrix. The search for a negative eigenvalue occurs by iteration using the Lanczos algorithm. In this approach, at each iteration, Lanczos calls VASP code to calculate the atomic forces from different configurations (Figure 1). The use of DFT increases the physical accuracy during the Minimum Energy Path (MEP) search and allows atomic scale chemical reaction to occur, generating a reliable energy landscape description even for transition points. The MEP search procedure can be summarized as follows: from a given relaxed state obtained by VASP configuration optimization with VASP, ARTn searches for a MEP, where forces of each step along the MEP are estimated from a single point calculation using VASP. Once the saddle point is obtained, the configuration is relaxed into a new minimum using VASP. Due to ARTn's efficiency, only a few hundred force evaluations are necessary to generate an event, i.e., converging to a saddle point from a local minimum, and relaxing into a new nearby state [36]. 


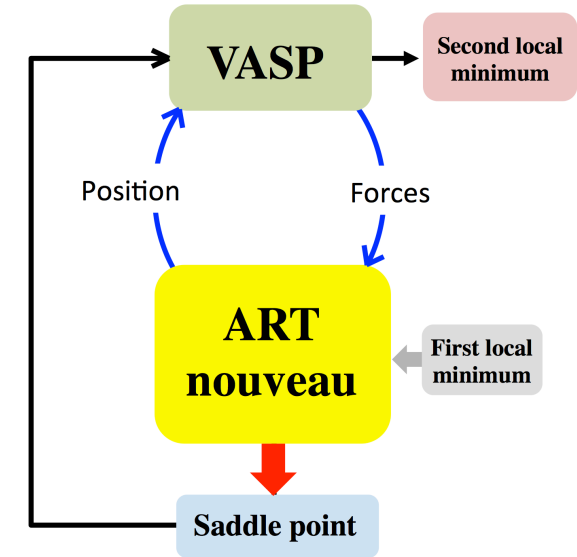

Figure 1: ARTn-VASP coupling procedure.

\subsection{Details of DFT calculations}

The generalized gradient approximation of Perdew-Burke-Ernzehof is used as exchange and correlation energy functional [45]. We use PAW pseudopotentials for the silicon, oxygen and hydrogen atoms $[46,47]$. The plane wave energy cut-off is fixed at $400 \mathrm{eV}$ and the $\Gamma$ point is used to sample the Brillouin zone. The spin polarization is taken into account to describe adequately the oxygen molecule and its spin conversion. In all our calculations, the oxygen molecule is free to relax and the spin is free to convert.

\subsection{Simulation cell}

The $\mathrm{Si}(100)$ surface is modeled as a repeated $\mathrm{Si}_{224} \mathrm{H}_{32}$ slab with a $p(2 \times 2)$ reconstruction on the surface. The cell is formed with fourteen layers of sixteen silicon atoms (Figure 2a). The surface contains eight dimer units. Hydrogen atoms are placed to passivate the dangling bonds of the silicon atoms below the slab. The seven bottom layers of the slab and hydrogen atoms are kept fixed to simulate the bulk, all other atoms are free to relax. A vacuum zone of $15 \AA$ is introduced above the surface.

The objective of this study is to investigate the thermal oxidation process beyond the oxidation of the silicon topmost layer. The oxidized surface model has been constructed as reported in the literature and based on our previous results $[16,17,43,44]$. We modeled partially and fully oxidized surfaces by adsorbing sixteen or thirty two oxygen atoms respectively, using an elementary pattern that can be depicted as alternate 4-O atom and 6-O atom rings as shown on Figure $2 \mathrm{~b}$ and Figure 3. This atomic scheme is observed as a favorable oxidized configuration for the silicon surface in several works and represents a well-characterized starting point $[21,44,48,49]$. In the following, we label the subsurface area between the uppermost oxidized layer and atomic layers below as surface-1, surface- 2 and 
surface-3 layers, following Figure 2f. The atomic positions for these initial configurations are directly derived from the output of the calculations described in our previous papers $[16,17]$, where 4-O-atom ring is characterized by an Adjacent Dimer Bond (ADB) as described in Ref 16. Considering our slab size, the partially oxidized surface contains two 6-O atom rings (Figure $2 \mathrm{~b}$ and Figure $3 \mathrm{a}$ ) and two 4-O atom rings and the fully oxidized surface contains four 6-O atom rings and four 4-O atom rings (Figure $3 \mathrm{~b}$ and c). In the following, we use 4/6 $\mathrm{O}$ ring pattern to refer to alternate 4-O atom and 6-O atom rings.
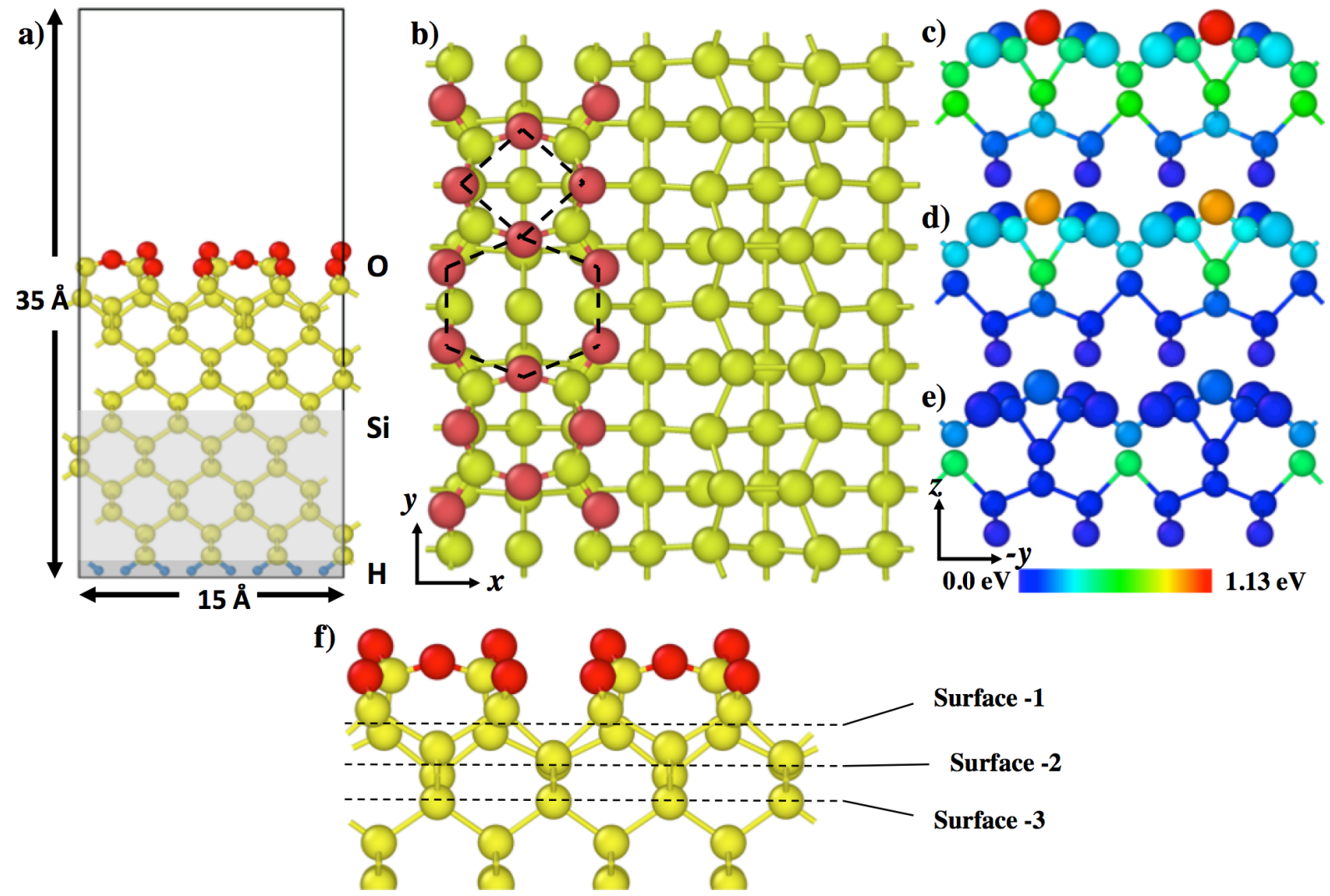

Figure 2: (a) Side view (xz plane) of simulation cell $\left(\mathrm{Si}_{224} \mathrm{H}_{32} \mathrm{O}_{32}\right)$. The topmost silicon atoms (yellow spheres) of the slab are fully oxidized (oxygen atoms in red spheres). (b) Top view of the $\mathrm{Si}(100)$ surface where only one row of dimers is oxidized: on the right the $(2 \times 1)$ reconstruction on the row of dimers is visible and on the left the dotted black lines highlight the alternating 6-O atom and 4-O atom rings used as the elementary pattern in this study. The dashed square corresponds to the 4-O ring and the dashed hexagon to the 6-O ring. The adsorption site is located into the 6-O rings. (c), (d) and (e) are side views (yz plane) with a color range used to quantify the total strain energy per atom in (c) calculated using eq. 2.

This total strain energy can be decomposed as angular distortion (shown in (d)) and stretches strain energy (shown in (e)) contributions. On (c) (d) and (e), the larger spheres correspond to oxygen atoms and smaller ones refer to silicon atoms. $x, y$ and $z$ directions correspond to [110], [-110] and [001] respectively.

(f) is a side view of the silicon slab where the description of subsurface layers used in the paper is provided. 


\subsection{Simulation of the adsorption reaction of oxygen molecule}

The alternate $4 / 6 \mathrm{O}$ ring elementary pattern used as an oxidized surface model exhibits only few reactive sites for the adsorption of a new oxygen molecule: on this surface, several adsorption sites as a function of surface topology were investigated (not detailed here), and only the 6-O ring appears (dashed hexagons on Figure $2 \mathrm{~b}$ and 3 ) as a reactive site as it is wide enough to allow adsorption and further insertion of dioxygen molecule into the surface.

For this study we proceed as follows: initially a dioxygen molecule is positioned at $2 \AA$ above a 6-O ring adsorption site and its configuration relaxed with VASP. Adsorption of one to two dioxygen molecules is performed in each adsorption 6-O ring site to simulate increasing coverage on the partially oxidized surface. Adsorption of one to four dioxygen molecules is performed in each adsorption 6-O ring site on the fully oxidized surface to simulate increasing coverage on fully oxidized surface (See Figure 3, due to the periodic condition only one 6-O ring adsorption site on Configuration $\mathrm{A}$ and two 6-O ring adsorption sites are visible on the configurations $\mathrm{B}$ and $\mathrm{C}$ ). Atomic diffusion into the interfacial subsurface area from each resulting configuration is then analyzed with ARTn-VASP.

\subsection{Adsorption energies and strain energy calculations}

The adsorption energy of dioxygen molecule in section 3.2 is calculated according to:

$$
E_{\text {ads }}=E_{\mathrm{O}_{2} \text { adsonoxmodel }}-\left(E_{\text {oxmodel }}+E_{\mathrm{O}_{2}}\right)
$$

where $E_{\mathrm{O}_{2}}$ adsonoxmodel corresponds to the total energy of the adsorbed oxygen molecule on the oxidized surface model, $E_{\text {oxmodel }}$ is the total energy of the oxidized surface model containing sixteen or thrity two oxygen atoms depending on the considered partially and totally oxidized surface model respectively and $E_{O_{2}}$ the energy of an isolated oxygen molecule.

In the following we discuss the variation of the total strain energy of the system and the atomic strain energy for local characterization. For convenience we use the Valence Force Field (VFF) approach $[53,55]$ that considers the variation of the total energy through two contributions: one comes from the variation of the suboxide energy $\left(\Delta E_{\text {subox }}\right)$ and the second part is related to the variation of the strain energy variation $\left(\Delta E_{\text {strain }}\right)$. The Keating formalism [51] is used to evaluate the local strain and has been widely used for study the $\mathrm{SiO}_{2} / \mathrm{Si}(001)$ interface [52-56]. The strain energy $\left(\mathrm{E}_{\text {strain }}\right)$ is taken to be zero for perfect structure (Si bulk or $\mathrm{SiO}_{2}$ bulk) and positive for any deviation from the perfect structure: 


$$
E_{\text {Strain }}=\frac{1}{2} \sum_{i} k_{b}\left(b_{i}-b_{0}\right)^{2}+\frac{1}{2} \sum_{i, j} k_{\theta}\left(\cos \theta_{i j}-\cos \theta_{0}\right)^{2}
$$

where $k_{b}$ (in $\mathrm{eV} / \AA^{2}$ ) and $k_{\theta}$ (in $\mathrm{eV}$ ) are constants associated with bond stretching and angle distortion respectively. $b_{i}$ and $b_{0}$ (in $\AA$ ) represent the bond length of $i$ th bond and its equilibrium distance, and $\theta_{i j}$ and $\theta_{0}$ the angle between the $i$ th and $j$ th bonds and its equilibrium angle, respectively. The Keating parameters used for Eq. 2 are issued from the Table I (KT(sh) parameters) in Ref. 55 where these data are fitted on the energy calculated using DFT calculation for the $\mathrm{Si} / \mathrm{SiO}_{2}$ interface after removing the suboxide part. In this model, we do not consider the oxygen-oxygen bond. The total strain energy is obtained by summing over all bonds. In the following, we use $\Delta \mathrm{E}_{\text {strain }}$ as the $\mathrm{E}_{\text {strain }}$ difference between two configurations.

An atomic strain energy can be defined and assigned to each atom by projecting half of each bond contribution onto a given $\mathrm{O}$ or $\mathrm{Si}$ atom. This atomic strain energy parameter is used throughout the paper to help understand the propagation front of the oxidation process. These values are put in relation with activation barrier changes in the results section (sections 3.3 and 4). In Figure 2c, the estimated strain concentration in the three topmost layers at the interface is shown. By decomposing the total strain energy, two strained domains appear: a first one where the strain is dominated by the angular distortion, due to the angle on the silicon atom beneath the ADB close by $-42.0^{\circ}$ compared to $109.47^{\circ}$ (tetrahedral angle) and to the angle $\mathrm{Si}-\mathrm{O}-\mathrm{Si}$ formed by the ADB in the $4-\mathrm{O}$ ring which decrease to $-82.1^{\circ}$ compared to the equilibrium angle $\left(180^{\circ}\right)$ (Figure $2 \mathrm{~d}$ ) and a second domain where the strain is due to the $\mathrm{Si}-\mathrm{Si}$ bond stretching (Figure $2 \mathrm{e}$ ) beneath the 6-O ring in the row of dimers $(\mathrm{d}(\mathrm{Si}-\mathrm{Si})=2.49$ $2.50 \AA$, compared to $2.36 \AA$ in the silicon bulk). The identified 6-O ring adsorption site in this structure corresponds to a tensile strain domain.

The ARTn-VASP MEP search procedure is performed to investigate the diffusion mechanism of the oxygen atom at the interface. In spite of the large number of events generated, we cannot assume a complete set, however, previous experience with ARTn and analysis of the found mechanisms suggest that we identify some major diffusion pathways. To facilitate reading, we focus here only on typical mechanisms-associated with the propagation of the oxidation front at the $\mathrm{Si} / \mathrm{SiO}_{2}$ interface, the full list is available upon request.

\section{ATOMIC INSIGHT OF THE PROPAGATION OF THE OXIDATION FRONT AT THE $\mathrm{Si}_{\text {/SiO }}$ INTERFACE: ADSORPTION AND SUBSEQUENT DIFFUSIONS}




\subsection{Initial strain in oxidized surface-models}

a) Configuration $A$

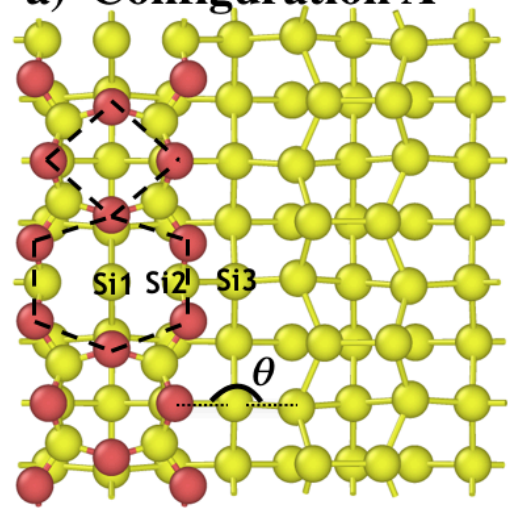

b) Configuration B

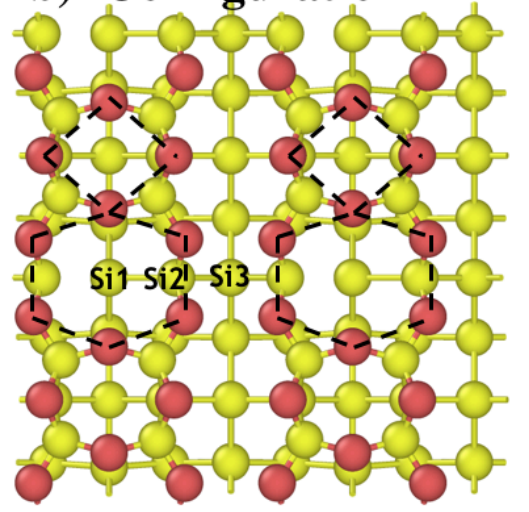

c) Configuration $\mathbf{C}$

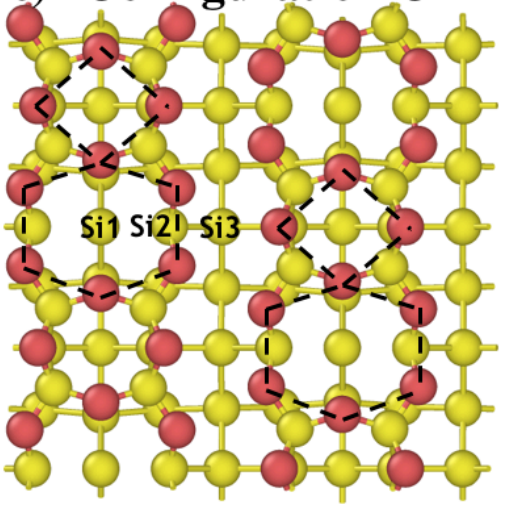

Figure 3: Top views of the three surface oxidation configurations used here and exhibiting the elementary 40/60 pattern as described in Figure $2 \mathrm{~b}$. a) The surface is half covered using two elementary patterns on

a single row of dimers, b) and c) the silicon surface is fully oxidized arranged symmetrically and

antisymmetrically compared to the channel axis respectively. Structures b) and c) have energy difference of $-0.95 \mathrm{eV}$ in favor of c). At the interface, we identify $\mathrm{Si}$, Si2 and $\mathrm{Si3}$ silicon atoms: Si1 is the silicon atom below the row of dimers in the middle of the 6-O ring in the surface-2 layer, $\mathrm{Si} 2$ is the silicon atom on the side of the 6-O ring in the surface-1 layer and $\mathrm{Si3}$ is the silicon atom in the channel in the surface-2 layer.

To study the environment and local strain effect on diffusion mechanisms, three surface coverage models are used as shown in Figure 3. These surfaces are distinguishable by their $6 / 4 \mathrm{O}$ ring pattern distribution on the surface. A low coverage is modeled with only one oxidized row of dimers (configuration A) and two fully oxidized surfaces are considered where the two row of dimers can be oxidized with a symmetric 6/4 $\mathrm{O}$ ring pattern distribution considering the channel axis (configuration B) and antisymmetric distribution (configuration C). In the following, local structure deformation in each configuration is discussed by providing the atomic strain energy on key atoms from the interface.

Oxidized or not, rows of dimers are connected by a silicon atom in the channel called $\mathrm{Si} 3$ in Figure 3. The strain on the $\mathrm{Si} 3$ atom depends on whether $\mathrm{Si} 3$ is connected to a 6-O ring, a 4-O ring or to a non-oxidized row. This strain is related to the angular deformation associated with the ring size: in configuration $\mathrm{A}$, the strain energy on 6-O-ring $\mathrm{Si} 3$ is only $0.02 \mathrm{eV}$ with $\theta=$ $105.5^{\circ}$ while it is four times higher, at $0.08 \mathrm{eV}$ with $\theta=123.8^{\circ}$ on a Si3 linked to the 4-O ring. A similar but less important strain difference is observed in configuration B with the strain energy going from 0.10 to $0.12 \mathrm{eV}$ between the two rings. In this configuration, the juxtaposition of the same types of rings emphasizes the compressive effect on the environment of $6-\mathrm{O}$ ring with $\theta=93.8^{\circ}$ by opposition to the tensile effect of 4-O ring with $\theta$ 
$=128.3^{\circ}$. Because of symmetry, no strain difference is observed for Si3 between the two rings in configuration $\mathrm{C}$ : in both cases, the strain energy is $0.04 \mathrm{eV}$ with $\theta=112.1^{\circ}$, close to the tetrahedral equilibrium angle $\left(\theta_{0}=109.5^{\circ}\right)$.

Elastic deformation can also take place through bond stretching below the row of dimers on $\mathrm{Si1}-\mathrm{Si} 2$ and Si2-Si3 between surface-1 and surface-2 layers. Si1-Si2 and Si2-Si3 bond lengths are $2.56 \AA$ and $2.37 \AA$ respectively in configurations $\mathrm{A}$ and $\mathrm{C}$ and $2.41 \AA$ and $2.31 \AA$ in configuration $\mathrm{B}$, which can be explained by the compressive effect of 6-O ring, more important in B than in A and C. Thus, the main contribution of the stretch deformation in the strain energy comes from the Si-Si bonds located below the row of dimers.

\subsection{Adsorption of $\mathrm{O}_{2}$ molecule}

As mentioned previously in section 2.5, adsorption of oxygen molecules is conducted on the 6-O ring site as it appears to be the only reactive site at large coverage. Assuming this, we investigate two successive molecular oxygen adsorptions on the two 6-O rings of configuration $\mathrm{A}$ and four successive $\mathrm{O}_{2}$ adsorptions on the four 6-O rings of configurations $\mathrm{B}$ and $\mathrm{C}$. Adsorptions on $\mathrm{B}$ and $\mathrm{C}$ are conducted by sequentially filling one row of dimers before oxidizing the second row.

Throughout this paper we use the following notation: A1 and A2, B1, B2, B3 and B4 and C1, $\mathrm{C} 2, \mathrm{C} 3$ and $\mathrm{C} 4$ to refer to increasing $\mathrm{O}_{2}$ deposition on a given configuration. A1 corresponds to the structure obtained after a single adsorption on configuration A, B3 is the adsorbed configuration after the deposition of three oxygen molecules on starting configuration $\mathrm{B}$, and $\mathrm{C} 2$ the adsorbed configuration after deposition of two oxygen molecules on starting configuration $\mathrm{C}$.

After each deposition, the oxygen molecule penetrates beneath the surface oxidized layer through the 6-O ring and oxidizes the $\mathrm{Si}-\mathrm{Si}$ bonds below the row of dimers between the surface-1 and the surface-2 layers. Adsorption energies $\left(\mathrm{E}_{\mathrm{ads}}\right)$ and variations of strain energies calculated considering all the atoms of the simulation cell $\left(\Delta \mathrm{E}_{\text {strain }}\right)$ are reported in Table 1 . The adsorption $v s$. variation of strain energies are plotted in Figure 4a and the corresponding configurations in Figure $4 \mathrm{~b}$. The plot clearly shows a linear relationship between these two energies. This result validates the used approach that consists in the separation of total energy in two contributions: the energy of the suboxide and the strain energy. Here, the variation of the suboxide energy during the adsorption is $-8.36 \mathrm{eV}$ and the strain energy contributes to the total energy with a proportional coefficient calculated to be 0.46 . 
These adsorbed configurations are the starting configurations for interfacial diffusion in the surface-2 layer and toward the deeper layer resulting in the oxidation of the $\mathrm{Si}-\mathrm{Si}$ bond between surface- 2 and surface-3 layers, i.e. at the growing $\mathrm{Si} / \mathrm{SiO}_{2}$ interface.

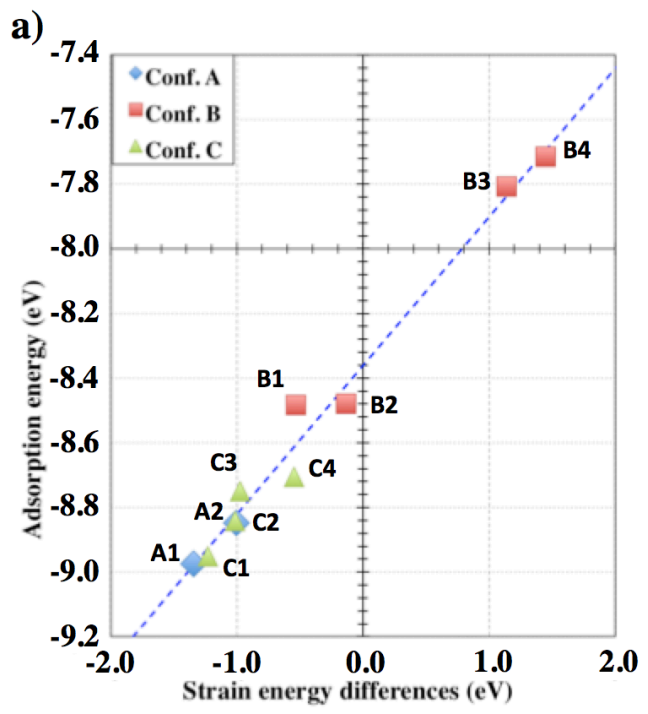

b)

A1

B1

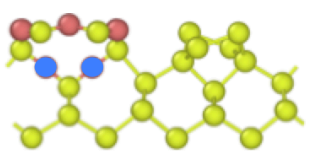

B3

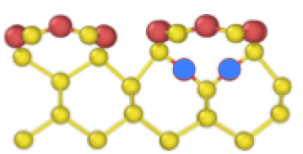

C1
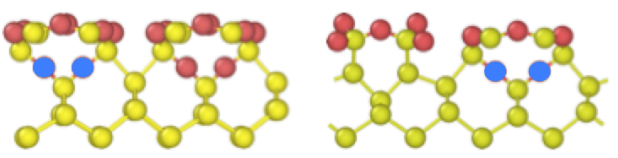

Figure 4: a) Adsorption energies as function of strain energy change due to oxygen molecule adsorption.

Values are given in Table 1. The fit corresponding to the dashed line is $y=0.46 x-8.36$. b) Side view in the (xz) plane of selected different configurations to be analyzed with ARTn-VASP coupling where the number associated to the configuration letter indicates the number of adsorbed additional oxygen molecules. In blue the new adsorbed oxygen atoms are highlighted.

Table 1: Strain energies changes $\left(\Delta E_{\text {strain }}\right)$ induced by oxygen molecule adsorption calculated according to Eq. 2, and oxygen molecule adsorption energies $\left(E_{\text {ads }}\right)(E q .1)$ for selected adsorbed configurations. Some of these configurations are shown on Figure $4 \mathrm{~b}$.

\begin{tabular}{c|c|c} 
& $\boldsymbol{\Delta} \mathbf{E}_{\text {strain }}(\mathbf{e V})$ & $\mathbf{E}_{\text {ads }}(\mathbf{e V})$ \\
\hline $\mathbf{A 1}$ & -1.34 & -8.97 \\
\hline $\mathbf{A 2}$ & -1.00 & -8.85 \\
\hline
\end{tabular}




\begin{tabular}{c|c|c}
\hline B1 & -0.53 & -8.48 \\
\hline B2 & -0.14 & -8.48 \\
\hline B3 & 1.45 & -7.72 \\
\hline B4 & 1.14 & -7.81 \\
\hline C1 & -1.23 & -8.95 \\
\hline C2 & -1.02 & -8.84 \\
\hline C3 & -0.97 & -8.75 \\
\hline C4 & -0.55 & -8.71 \\
& &
\end{tabular}

Comparing configurations $\mathrm{A}$ and $\mathrm{C}$, we note that the increase in oxygen coverage decreases the overall configuration strain thanks to oxygen atoms that insert into the $\mathrm{Si}-\mathrm{Si}$ bonds beneath the row of dimers: the Si-Si bonds that are initially strongly stretched (2.50-2.56 depending on the considered configuration compared to the $2.37 \AA$ in bulk silicon) relax thanks to the formation of Si-O bonds of $1.63 \AA$, close to the characteristic Si-O bonds of silica (1.61 $\AA$ ). Atomic strain energy calculations reveal that stretching contribution surrounding atom Sil below the row of dimers decreases from $79 \%$ to $0 \%$ when evolving from $\mathrm{Si}-\mathrm{Si}$ bond to $\mathrm{Si}-\mathrm{O}$ bond on both configurations $\mathrm{A}$ or $\mathrm{C}$. Same tendency has been observed for $\mathrm{Si} 2$ from $40 \%$ to $10 \%$. Furthermore, the oxygen adsorption widens the 6-O ring.

For configuration $\mathrm{B}$, the silicon atom in the channel (Si3) and below the row of dimers (Si1) becomes even more compressed at each adsorption with the atomic strain energy increasing at each adsorption. This tendency is reflected in total strain energy given in Figure 4a where it increases with coverage. This shows how the strain is allocated along the silicon-oxygen chain with maximum strain variation on Si1 with the change of local environment while Si2 strain energy remains stable.

\subsection{Activated $\mathrm{O}$ diffusion at the $\mathrm{SiO}_{2} / \mathrm{Si}$ interface}

We now focus on the oxygen atom diffusion starting from four selected configurations $-\mathrm{A} 1$, $\mathrm{B} 1, \mathrm{~B} 3$ and $\mathrm{C} 1$, looking more specifically at atomistic mechanisms enabling the diffusion 
front to progress. These four configurations have been chosen since they differ significantly in terms of energy barriers and energy gains. Since mechanisms obtained from A1 and A2, B1 and $\mathrm{B} 2, \mathrm{~B} 3$ and $\mathrm{B} 4$ or $\mathrm{C} 1$ to $\mathrm{C} 4$ are energetically very similar, we chose not to repeat the discussion for diffusion from A2, B2, B4 and C2, C3, and C4. Among the diffusion pathways identified by ARTn, we leave aside about 10 local mechanisms characterized by too high activation barriers or local mechanisms leading to final states less stable than the initial state, irrespective of the coverage. Note that a mechanism not favored at low coverage can become favorable with increasing coverage and vice versa.

Diffusion pathways are investigated as a function of oxygen coverage. For clarity, we discuss only mechanisms that enable the oxidation front to propagate, i.e., those associated with the lowest activation barriers or that allow the system to stabilize. For the selected diffusion pathways, we establish a correlation between the activation barriers and energy gains along the diffusion pathway and the strain energy accumulated in the substrate.

In Figure 5, we present $\mathrm{O}$ diffusion mechanisms at the interface obtained with ARTn-VASP from configurations $\mathrm{A} 1, \mathrm{~B} 1, \mathrm{~B} 3$ and $\mathrm{C} 1$. The configurations obtained after $\mathrm{O}$ diffusion in these structures are provided in Figure 5a and a schematic view of these diffusion pathways is given in Figure 5b. The same final structural configurations obtained from the different starting configurations are referred by the same unique tag in Figure 5a. For instance, the final configuration \#2 can be obtained from A1, B1 and B3, independently of the coverage and will be designed as A1-\#2, B1-\#2 and B3-\#2 respectively. To be consistent with this notation, the diffusion mechanism leading to such final configurations will be referred to as diffusion mechanism \#2. Same rules are applied to mechanisms \#1 and \#3.

These three favorable diffusion pathways involve predominantly either Si or O (Figure 5b). Mechanism \#1 can be described as the diffusion of a silicon atom into the surface-2 layer resulting in a reconstruction at the interface, with the formation of a Si-Si dimer. In \#2, the oxygen atom located beneath the 6-O ring between surface-1 and -2 layers oxidizes the silicon atom in the channel, resulting in an oxidized Si-Si dimer at the interface through formation of a $\mathrm{Si}-\mathrm{O}$ bond with the $\mathrm{Si} 3$ atom. In \#3, an oxygen atom diffuses deeper into the bulk starting from between surface- 1 and surface- 2 layers to end between surface- 2 and surface- 3 layers.

The various energy variables associated with these diffusion mechanisms (activation energy $\left(\mathrm{E}_{\mathrm{ac}}\right)$ and relative stability $(\mathrm{dE})$ calculated as the difference of the total energy between the initial state and the final state of the diffusion mechanism) are given in Table 2. $\mathrm{E}_{\mathrm{ac}}$ ranges from a relatively low 0.63 to $3.42 \mathrm{eV}$, corresponding to an unlikely diffusion at usual 
deposition temperatures. Negative values for $\mathrm{dE}$ indicate stabilization of the system. Note that when $\mathrm{dE}$ is close to 0 , the two configurations have the same probability to exist, from a thermodynamical point of view. For most events, the global atomic displacement perpendicular to the surface $(\mathrm{dz})$, calculated as the sum of the displacement of all the atoms along the $z$-axis, is positive, which indicates a surface swelling up as atoms diffuse towards the bulk.
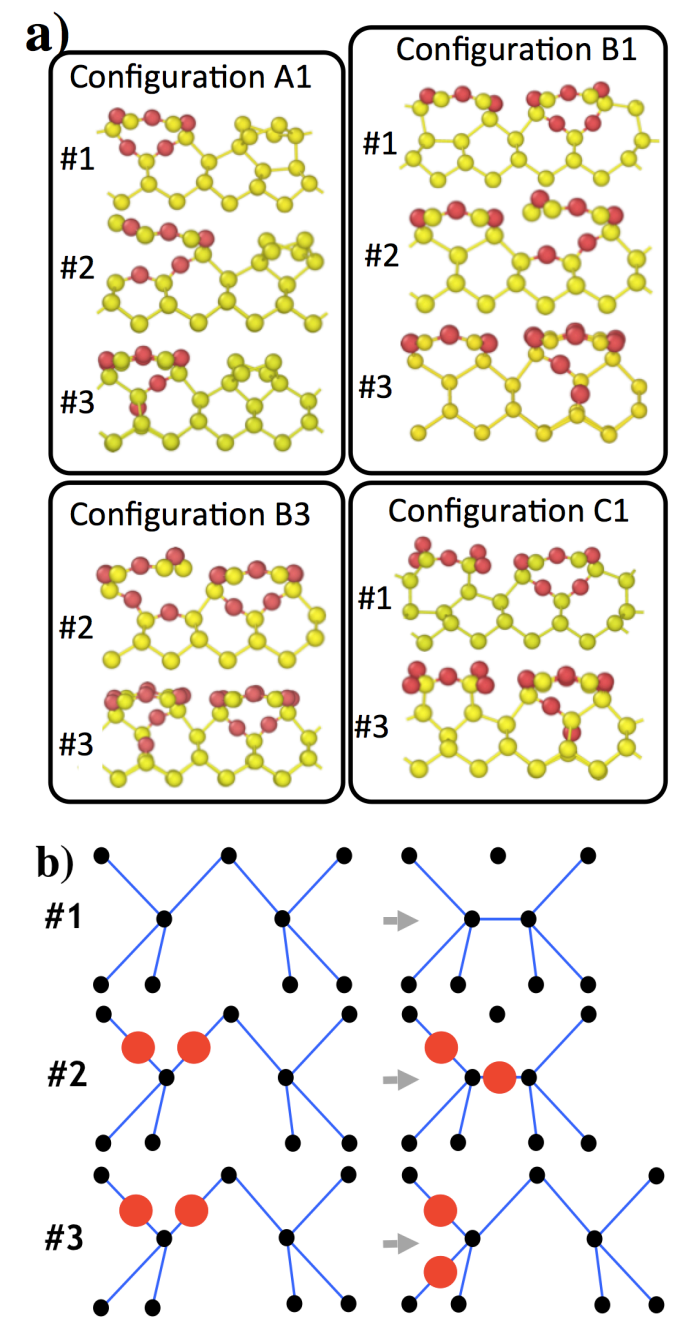

Figure 5: a) Final configurations obtained with ARTn-VASP coupling obtained from initial configurations A1, B1, B3 and C1. Same final structural configurations obtained from different starting configurations are referred to using the same label. A1, B1, $\mathrm{C} 1$ and $\mathrm{B} 3$ do not correspond to the same oxygen coverage. $\mathrm{b}$ ) Schematic representation of three described diffusions obtained with ARTn-VASP coupling.

Table 2: Mechanisms obtained with ARTn-VASP coupling from configurations A1, B1, B3 and C1. Numbers correspond to events presented in Figure 5. For each mechanism, we show the activation energy $\left(E_{a c}\right)$, the relative stability $(\mathrm{dE})$ and the total displacement perpendicular to the surface along $z$-axis of the event $(\mathbf{d z})$. 


\begin{tabular}{|l|c|c|c|}
\hline Event & $\mathrm{E}_{\mathrm{ac}}(\mathrm{eV})$ & $\mathrm{dE}(\mathrm{eV})$ & $\mathrm{dz}(\mathrm{A})$ \\
\hline A1-\#1 & 1.21 & 0.17 & 3.06 \\
\hline A1-\#2 & 1.46 & 0.87 & 3.98 \\
\hline A1-\#3 & 2.96 & 0.49 & -0.52 \\
\hline B1-\#1 & 3.42 & -0.80 & 4.54 \\
\hline B1-\#2 & 0.89 & 0.50 & 3.82 \\
\hline B1-\#3 & 2.77 & 0.20 & -0.60 \\
\hline B3-\#2 & 0.63 & -0.28 & 4.22 \\
\hline B3-\#3 & 1.80 & 0.04 & -0.77 \\
\hline C1-\#1 & 2.57 & 1.39 & 2.89 \\
\hline C1-\#3 & 3.22 & 0.49 & -1.27 \\
\hline
\end{tabular}

It is interesting to detail a few of these events. From A1, corresponding to a low coverage in oxygen species, both pathways \#1 and \#2 involve accessible barriers with an advantage for $\# 1$, with $\mathrm{E}_{\mathrm{ac}}=1.21 \mathrm{eV}$. The same mechanism \#1, starting from $\mathrm{B} 1$ and $\mathrm{C} 1$ and leading to configurations $\mathrm{B} 1-\# 1$ and $\mathrm{C} 1-\# 1$, is less probable, associated with significantly higher activation energies $\left(\mathrm{E}_{\mathrm{ac}}=3.42 \mathrm{eV}\right.$ and $2.57 \mathrm{eV}$ respectively). Since configuration A1-\#1 shows a total energy close to that of the initial state $(\mathrm{dE}=0.17 \mathrm{eV})$, the interface reconstruction by $\mathrm{Si}-\mathrm{Si}$ dimer creation is thus a favorable mechanism at low coverage.

For the fully oxidized B1, even though it requires crossing high energy barrier, configuration B1-\#1 associated with the interface reconstruction in a non-oxidized area below the row of dimers appears clearly as the most thermodynamically favorable with an energy $-0.80 \mathrm{eV}$ below that of the initial state. By comparison, $\mathrm{B} 1-\# 2$, with $\mathrm{dE}=0.50 \mathrm{eV}$, is less stable but can be access more easily from a kinetic point of view with a $0.89 \mathrm{eV}$ barrier.

Note here that as coverage increases, the activation barrier of the pathway \#2 lowers, from A1 to $\mathrm{B} 1$ and to $\mathrm{B} 3$ barriers falls from 1.46 to 0.89 and $0.63 \mathrm{eV}$ respectively, while the final state gains in stability: with $\mathrm{dE}=-0.28 \mathrm{eV}$, for $\mathrm{B} 3-\# 2,0.78 \mathrm{eV}$ below $\mathrm{B} 1-\# 2$ and $1.08 \mathrm{eV}$ below A1- 
\#2. A similar trend is observed for pathway \#3, where B3-\#3 is more stable by $0.16 \mathrm{eV}$ with respect to B1-\#3, and for pathway \#1: $\mathrm{dE}=-0.80 \mathrm{eV}$ for $\mathrm{B} 1-\# 1$ and $0.17 \mathrm{eV}$ for $\mathrm{A} 1-\# 1$.

This suggests that an unlikely diffusion event at low coverage can become the dominant mechanism at the higher coverage, enabling the oxidation front to progress, as local strain evolves.

ARTn-VASP does not identify any mechanism from C1 that would lead to a configuration similar to configuration \#2. Generated pathways are rather characterized by high activation energies associated with a much slower kinetics.

These results suggest that oxygen coverage is a dominant factor for oxidation at the $\mathrm{Si} / \mathrm{SiO}_{2}$ interface: a configuration that is initially unfavourable energetically, becomes favored at higher coverage. This observation confirms what had already been seen at very low coverage [16]: the silicon surface adapts to the presence of oxygen species. At low coverage, silicon atoms are likely to diffuse at the interface leading to interface modification during the growth; $\mathrm{Si}-\mathrm{Si}$ bonds, that are weaker than $\mathrm{Si}-\mathrm{O}$ bonds, break first to reconstruct the interface. As the surface becomes fully oxidized, oxygen atom diffusion is favored due to the accumulated strain.

\section{DISCUSSION: STRAIN-DRIVEN DIFFUSION PROCESS}

We can correlate the various diffusion mechanisms with the evolution of the strain energy in the system. This correlation is presented for mechanism \#2 (A1-\#2, B1-\#2 and B3-\#2) in Figure 6 and for mechanism \#3 (A1-\#3, C1-\#3, B1-\#3 and B3-\#3) in Figure 7.

Figure 6 describes the mechanism \#2 associated with an oxygen diffusing in surface-2 layer from structures A1, B1 and B3 (up). The atomic strain energy is highlighted at the saddle point (middle) and for the final configurations A1-\#2, B1-\#2 and B3-\#2 (down) as a difference with the respective starting structures. 


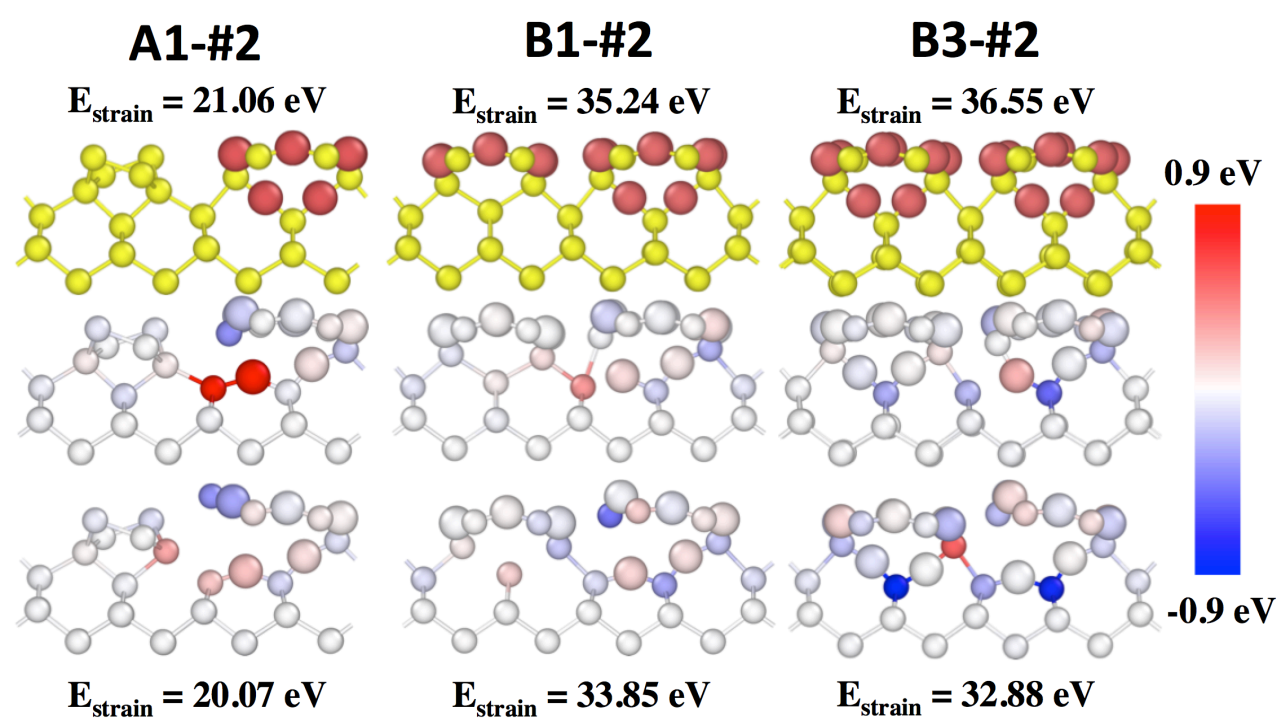

Figure 6: Diffusion path \#2 from A1 (left), B1 (middle) and B3 (right). From top to bottom, initial configuration, the saddle point and final state are provided. The color for saddle point and final configuration represent the atomic strain energy difference with initial configuration calculated with eq.

2. The strain energy ranges from $-0.90 \mathrm{eV}$ to $0.90 \mathrm{eV}$ and its color scale is given on the right. The total strain energy of the initial and final configurations are given above and below the structures. The oxygen atoms are represented in red or by large spheres and silicon atoms in yellow or by little spheres.

As discussed in the previous section, the activation energy decreases as the oxygen coverage increases and the sub-surface oxidation progresses (Table 2). The final state in A1 with the $\mathrm{Si} 2$ silicon atom pointing upward differs from the final states of B1 and B3 where the silicon atom points downward because of the presence of the 6-O ring in the vicinity. This explains the barrier, probably electrostatic, of around $0.20 \mathrm{eV}$ to flip silicon atom into a configuration where it points upward in the final states B1 and B3.

More crucially, however, this trend can be correlated with a reduced strain on the diffusing oxygen atom at the saddle point and on the silicon atom in the channel at final state. For the densest coverage, configuration $\mathrm{B} 3$, the $\mathrm{Si} 1$ silicon atom relaxes at the interface $\mathrm{Si} / \mathrm{SiO}_{2}$ as soon as diffusion is initiated, and only the diffusing oxygen exhibits a significant atomic strain energy. This occurs as the total strain energy goes up from $21.06 \mathrm{eV}$ for A1 to $35.24 \mathrm{eV}$ for $\mathrm{B} 1$ and $36.55 \mathrm{eV}$ for $\mathrm{B} 3$. In $\mathrm{B} 3$, the strain stored in the interfacial layer is such that an atomic rearrangement becomes necessary to relax the accumulated strain and reduce structural deformations. The presence of strain therefore lowers activation energies, facilitating diffusion and leading to the stabilization of the system. Stored strain energy, therefore, catalyzes $\mathrm{O}$ diffusion. 
This conclusion is supported by the similar trends observed for mechanism \#3 presented in Figure 7: as the total strain energy increases from $21.06 \mathrm{eV}$ in $\mathrm{A} 1$ to $34.29 \mathrm{eV}, 35.24 \mathrm{eV}$ and $36.55 \mathrm{eV}$ for $\mathrm{C} 1, \mathrm{~B} 1, \mathrm{~B} 3$ respectively, the activation energy decreases from $3.48 \mathrm{eV}$ in $\mathrm{A} 1$ to $3.22 \mathrm{eV}, 2.77 \mathrm{eV}$ and $1.80 \mathrm{eV}$, respectively while final states are stabilized with respect to the initial configurations with $\mathrm{dE}=0.52 \mathrm{eV}, 0.49 \mathrm{eV}, 0.20 \mathrm{eV}$ and $0.04 \mathrm{eV}$, respectively.
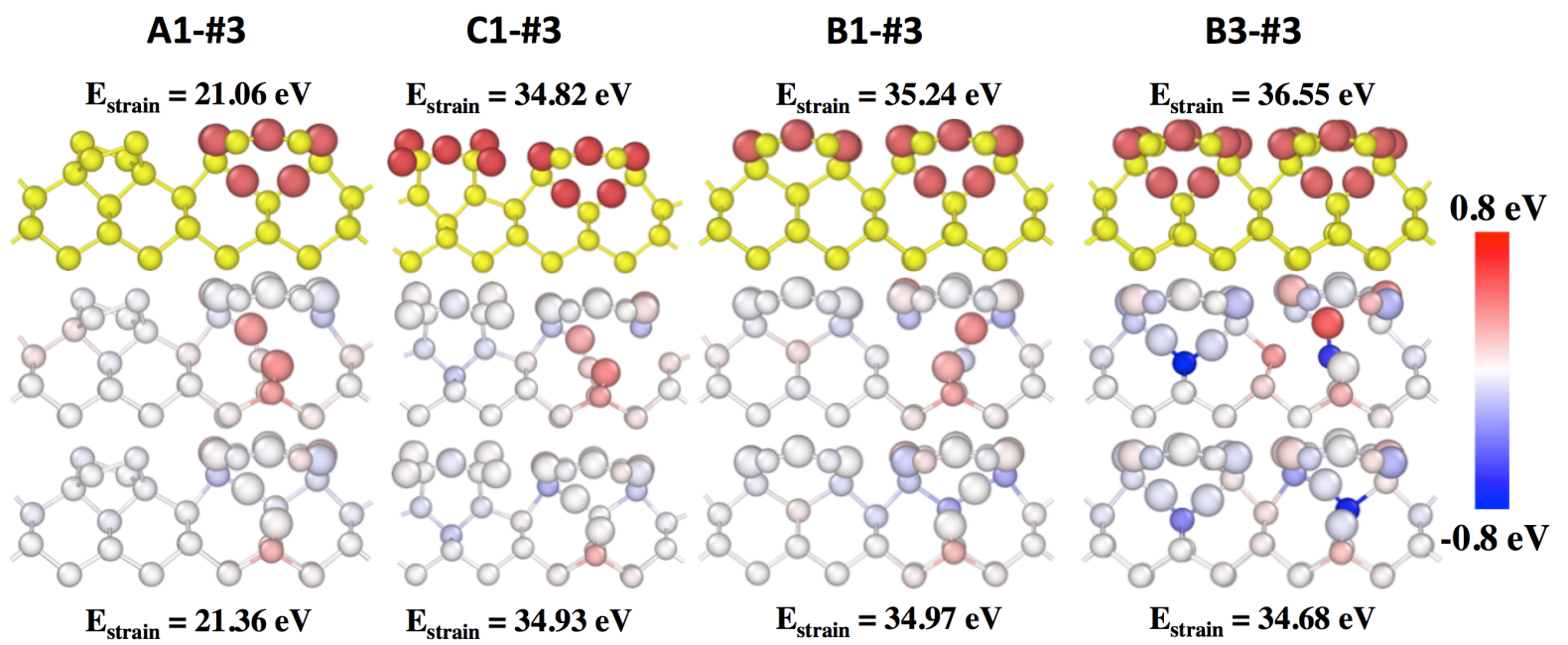

Figure 7: Left to right: Diffusion mechanism \#3 from A1, C1, B1 and B3 configurations, respectively.

From top to bottom, initial configuration, the saddle point and final state are provided. The colors for saddle point and final configuration represent the atomic strain energy difference with initial configuration calculated with eq. 2 . The associated strain energy ranges from $-0.80 \mathrm{eV}$ to $0.80 \mathrm{eV}$ and is colored according to the scale on the right. The total strain energy of the initial and final configurations are given above and below the structures. The oxygen atoms are represented in red or by large spheres and silicon atoms in yellow or by little spheres.

The activation energies and relative stabilities indicated in Table 2 are plotted in Figure 8 as a function of strain energy change $\left(\Delta \mathrm{E}_{\text {strain }}\right)$ between saddle (Eac) or final state $(\mathrm{dE})$ and initial state respectively. These values are intimately correlated to the initial strains and to their relaxation. The trend observed in Figure 8 is similar to the one observed between the adsorption energies and the strain energy changes shown in Figure 4a. 


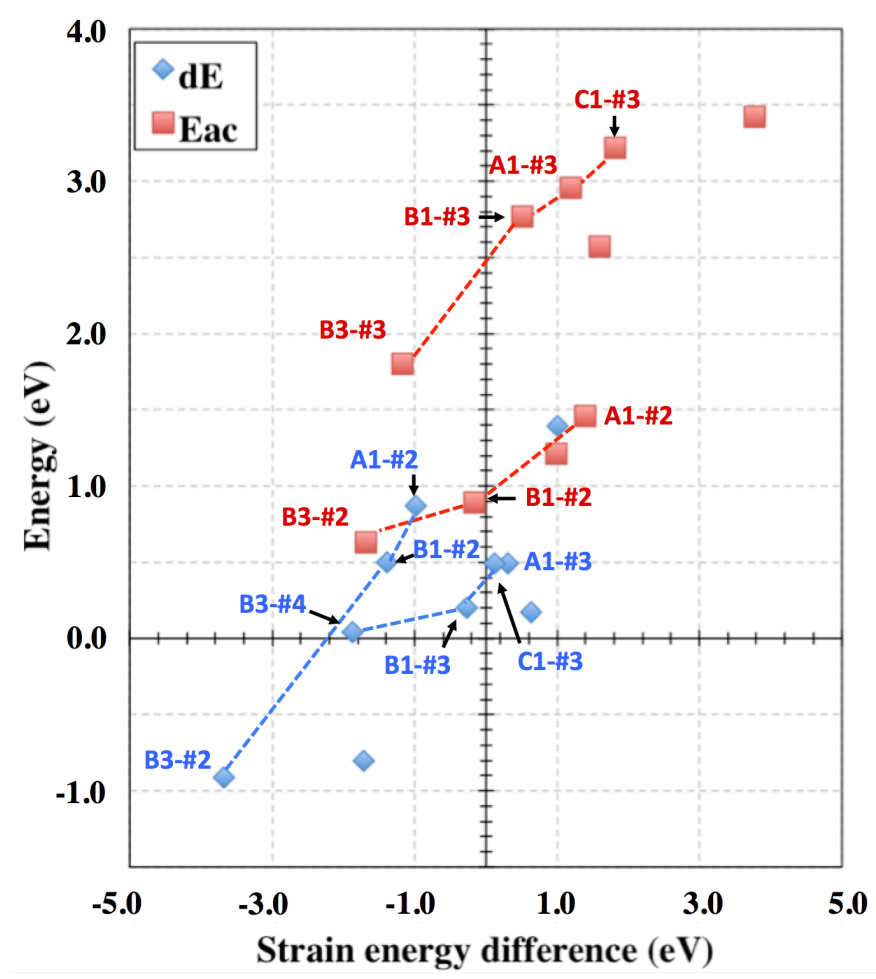

Figure 8: Correlation between the energetic parameters of diffusion mechanism as function of strain energy differences between initial and final configuration (dE) and initial and saddle point configuration (Eac) respectively. Dashed lines are a guide for the eye.

The strain, therefore, acts like an energy reservoir that can be drawn up during atomic diffusion and shifting the energy balance of various diffusion mechanisms. The higher the initial strain, the lowest $\mathrm{E}_{\mathrm{ac}}$ and $\mathrm{dE}$ become, favoring oxygen insertion. This accumulated strain is directly linked to the interfacial environment, i.e. to the oxygen coverage and to the oxidation state in sub-surface. We see, in particular, that the strain energy stored at the interface as the oxidation progresses, i.e for increasing coverage, drives the atomic diffusion. These results are consistent with a layer-by-layer growth mode during silicon thermal oxidation $[6,8]$.

No kinetically relevant diffusion mechanisms was observed from configuration C1. All identified pathways are associated with high activation barriers and energetically unfavorable final configurations. This illustrates the high stability of configuration $\mathrm{C}$ already discussed before. The 4-O and 6-O ring proximity on either sides of the channel reduces strain effects whereas configuration B increases them. However, while the correlation between strain building and layer-by-layer growth is clear for the systems studied here, it will be necessary to evaluate the effects at the large scale of oxide nanostructuring on the diffusion kinetics of 
oxidation front through mesoscopic modeling approach, such as kinetic Monte Carlo [15], while introducing the strain effect.

\section{CONCLUSION}

The oxidation mechanisms of sub-surface silicon (100) have been investigated using a coupling of ARTn-VASP methodologies. Different surface coverage with a 4/6 O ring pattern were studied in order to understand the effect of the local environment on oxygen adsorption and diffusion at the interface. The environment effect was discussed in terms of strain energy thanks to Keating model. The oxygen molecule adsorption energy shows a linear dependence with strain energy. Furthermore, the interface diffusions obtained exhibits also a close correlation with strain at the $\mathrm{Si} / \mathrm{SiO}_{2}$ interface as the oxidation progresses. We have shown that the interface reconstructions are rapidly favorable and that the oxidation of deep layers occurs when oxygen surface coverage is sufficiently important. The strain energy accumulated at the interface will favor atomic diffusions energetically at high coverage which were not allowed at low coverage. The strain accumulation in the structure decreases the activation energies and inverses the relative stability of final configurations. A progressive increasing strain in the system changing the trend and the feasibility of diffusion to occur at the $\mathrm{Si} / \mathrm{SiO}_{2}$ interface is consistent a layer-by-layer scheme of the silicon oxidation process.

The results presented here offer a clear picture for the first stage of oxidation: the built-up strain, as the surface oxidizes should accelerate the diffusion of oxygen. As the interface grows, however, the disordered $\mathrm{SiO}_{2}$ network should allow the strain to relax, which will slow the progression to a halt. This second phase remains to be characterized.

\section{Acknowledgments}

This work has been performed using CALMIP (GRANT 1552 and 1418), IDRIS (GRANT 100588) and Curie (GRANT gen7474) computer resources. We also thank Calcul Québec/Compute Canada for computer time. The project has been funded in part by the Axe Amérique du Nord from Université Paul Sabatier and Université de Montréal.

\section{References}

[1] R. G. Arns, Eng. Sci. Educ. J. 7, 233 (1998)

[2] T. Zhu and M. N. Chong, Nano Energy 12, 347 (2015)

[3] R. Jansen, Nat. Mater. 11 (May (5)) 400 (2012) 
[4] M. T. Bohr, R. S. Chau, T. Ghani and K. Mistry, IEEE Spectrum, October Issue (2007)

[5] J. H. Oh, H. W. Yeom, Y. Hagimoto, K. Ono, M. Oshima, N. Hirashita, M. Nywa, A. Toriumi and A. Kakizaki, Phys. Rev. B 63, 205310 (2001)

[6] S. Ogawa, A. Yoshigoe, S. Ishidzuka, Y. Teraoka and Y. Takakuwa, Jpn. J. Appl. Phys. 46, 3244 (2007)

[7] S. Ogawa and Y. Takakuwa, Jpn. J. Appl. Phys. 45, 7063 (2006)

[8] S. Ogawa, J. Tang, A. Yoshigoe, S. Ishidzuka, Y. Teraoka and Y. Takakuwa, Jpn. J. Appl. Phys. 52, 110128 (2013)

[9] S. Ogawa, J. Tang and Y. Takakuwa, AIP Adv. 5, 087146 (2015)

[10] H. Z. Massoud, J. D. Plummer and E. A. Irene, J. Electrochem. Soc. 132, 2685 (1985)

[11] E. Kobeda and A. Irene, J. Vac. Sci. Technol. B 7, 163 (1989)

[12] J. T. Fitch, C. H. Bjorkman and G. Lucovsky, Appl. Surf. Sci. 39, 103 (1989)

[13] A. Hemeryck, N. Richard, A. Estève and M. Djafari Rouhani, Surf. Sci. 601, 2082 (2007)

[14] A. Hemeryck, N. Richard, A. Estève and M. Djafari Rouhani, Surf. Sci. 601, 2339 (2007)

[15] A. Hemeryck, A. Estève, N. Richard, M. Djafari Rouhani and G. Landa, Surf. Sci. 603, 2134 (2009)

[16] A. Hemeryck, A. Estève, N. Richard, M. Djafari Rouhani and Y. J. Chabal, Phys. Rev. B. 79, 035317 (2009)

[17] A. Hemeryck, A. Mayne, N. Richard, A. Estève, Y.J. Chabal, M. Djafari Rouhani, G. Dujardin and G. Comtet, J. Chem. Phys. 126, 114707 (2007)

[18] N. Mousseau, L. K. Bélan, P. Brommer, J.-F. Joly, F. El-Mellouhi, E. Machado-Charry, M.-C. Marinica and P. Pochet, J. Atom. Mol. and Optic Phys. 2012, 14 (2012)

[19] T. Akiyama and H. Kageshima, Surf. Sci. 576, L65 (2005)

[20] L. Colombi and M. C. Payne, Phys. Rev. Lett. 95, 196101 (2005)

[21] T. Yamasaki, K. Kato and T. Uda, Phys. Rev. Lett. 91, 146102 (2003)

[22] A. Bongiorno and A. Pasquarello, Phys. Rev. Lett. 88, 125901 (2002)

[24] A. Bongiorno and A. Pasquarello, J. Mater. Sci. 40, 3047 (2005)

[25] K. Shiraishi, H. Kageshima and M. Uematsu, Jpn. J. Appl. Phys. 39, L1263 (2000)

[26] H. Kageshima and K. Shiraishi, Phys. Rev. Lett. 81, 5936 (1998)

[27] H. Watanabe, T. Baba and M. Ichikawa, Jpn. J. Appl. Phys. 39, 2015 (2000)

[28] T. Akiyama, T. Ito, H. Kageshima and M. Uematsu, Phys. Rev. B 77, 115356 (2008)

[29] R.J. Jaccodine and W.A. Schlegel, J. Appl. Phys. 37, 2429 (1966) 
[30] A. Korkin, J.C. Greer and G. Bersuker, Phys. Rev. B 73, 165312 (2006)

[31] X.L. Han, G. Larrieu and C.D. Krzeminski, Nanotech. 24, 495301 (2013)

[32] C.D. Krzeminski, X.L. Han and G. Larrieu, Appl. Phys. Lett. 100, 263111 (2012)

[33] P.F. Fazzini, C. Bonafos, A. Claverie, A. Hubert, T. Ernst and M. Respaud, J. Appl. Phys. 110, 033524 (2011).

[34] G. T. Barkema and N. Mousseau, Phys. Rev. Lett. 77, 4358 (1996)

[35] R. Malek and N. Mousseau, Phys. Rev. E 62, 7723-7728 (2000)

[36] E. Machado-Charry, L. K. Béland, D. Caliste, L. Genovese, N. Mousseau and P. Pochet, J. Chem Phys. 135, 034102 (2011)

[37] G. Kresse and J. Furthmüller, Phys. Rev. B 54, 11169 (1996)

[38] G. Kresse and J. Furthmüller, Phys. Rev. B 59, 1758 (1999)

[39] L. J. Munro and D. J. Wales, Phys. Rev. B 59, 3969 (1999)

[40] G. Henkelman and H. Jónsson, J. Chem. Phys., 111, 7010 (1999)

[41] N. Mousseau and G. T. Barkema, Phys. Rev. E 57, 2419 (1997)

[42] F. El Mellouhi, N. Mousseau and P. Ordejon, Phys. Rev. B 70, 205202 (2004)

[43] P. Ganster, L. K. Béland and N. Mousseau, Phys. Rev. B 86, 075408 (2012)

[44] F. Fuchs, W. G. Schmidt and F. Bechstedt, Phys. Rev. B 72, 075353 (2005)

[45] J.P. Perdew, K. Burke and M. Ernzerhof, Phys. Rev. Lett. 77, 3865 (1996)

[46] P.E. Blöchl, Phys. Rev. B 50, 17953 (1994)

[47] G. Kresse and J. Joubert, Phys. Rev. B 59, 1758 (1999)

[48] C. Kaneta, T. Yamasaki, T. Uchiyama, T. Uda and K. Terakura, Microelec. Eng. 48, 117 (1999)

[49] N. Takahashi, T. Yamasaki and C. Kaneta, Phys. Status Solidi B 251, 2169 (2014)

[50] K. Gaal-Nagy, A. Incze and G. Onida, Phys. Rev. B 79, 045312 (2009)

[51] P.N. Keating, Phys. Rev. 145, 637 (1996)

[52] K.O. Ng and D. Vanderbilt, Phys. Rev. B 59, 10132 (1999)

[53] Y. Tu and J. Tersoff, Phys. Rev. Lett. 84, 4393 (2000)

[54] Y. Tu and J. Tersoff, Phys. Rev. Lett. 89, 086102 (2002)

[55] S. Lee, R.J. Bondi and G. S. Hwang, J. Appl. Phys. 109, 113519 (2011)

[56] S. Lee, R.J. Bondi and G. S. Hwang, Phys. Rev. B 84, 045202 (2011)

\section{Supplementary Material}

The ARTn code with its interface with VASP is available from the authors as well as directly from the site http://normandmousseau.com 